\title{
Global search for right cell type as a treatment modality for cardiovascular disease
}

Type: Article

Abstract:

Introduction: Acute myocardial infarction is the primary cause of heart disease-related death in the world. Reperfusion therapy is currently the backbone of treatment for acute myocardial infarction albeit with many limitations. With the emergence of stem cells as potential therapeutic agents, attempts in using them to enhance cardiac function have increased exponentially. However, it has its own disadvantages, and we postulate that the primary drawback is choosing the right cell type and solving this may significantly contribute to ambitious goal of using stem cells in the regeneration medicine. Areas covered: This article discusses several types of stem cells that have been proven to be likely candidates for treating cardiovascular diseases and their uses up to date, focusing on their biological characterization and potential usage in preclinical and clinical work. Expert opinion: The research on cell therapies for cardiovascular disease is promising, but there is still much uncertainty surrounding the efficacy of these cells in clinical settings. With a wide range of cells available as potential treatment for cardiovascular diseases, one should avoid from being overzealous and extrapolating when reporting their data. Future studies should focus more in understanding the biological functions of the available cells lines.

\begin{tabular}{|c|l|}
\hline Author & Musa, S. ;Xin, L. Z.; ;Govindasamy, V. ;Fuen, F. W. ;Abu Kasim, N. H. \\
\hline Source & Expert Opinion on Biological Therapy \\
\hline ISSN & $1471-2598$ \\
\hline DOI & $10.1517 / 14712598.2014 .858694$ \\
\hline Volume (Issue) & $14(1)$ \\
\hline $63-73$ & $63-73$ \\
\hline Year & 2014 \\
\hline
\end{tabular}

Keyword:

cardiac regeneration;cardiomyocytes;myocardial infarction;stem cells;mesenchymal stem-cells;acute myocardial-infarction;randomized phase-1;trial;cardiac-function;functional-properties;signaling pathway;;progenitor cells;stromal cells;bone-marrow;ips cells;Biotechnology \& Applied Microbiology;Research \& Experimental MedicineURL:

- http://apps.webofknowledge.com search via Accession No >> 000328109400008

- Full text available at : $\mathrm{http} / / /$ informahealthcare.com/doi/abs/10.1517/14712598.2014.858694 